\title{
Targeting ROS1 rearrangements in non-small cell lung cancer with crizotinib and other kinase inhibitors
}

\author{
Kartik Sehgal, Rushad Patell, Deepa Rangachari, Daniel B. Costa \\ Department of Medicine, Division of Hematology/Oncology, Beth Israel Deaconess Medical Center, Harvard Medical School, Boston, MA, USA \\ Correspondence to: Daniel B. Costa, MD, PhD. Division of Hematology/Oncology, Beth Israel Deaconess Medical Center, Harvard Medical School, \\ 330 Brookline Av., Boston, MA 02215, USA. Email: dbcosta@bidmc.harvard.edu. \\ Comment on: Wu YL, Yang JC, Kim DW, et al. Phase II Study of Crizotinib in East Asian Patients With ROS1-Positive Advanced Non-Small-Cell \\ Lung Cancer. J Clin Oncol 2018;36:1405-11.
}

Submitted Jul 26, 2018. Accepted for publication Aug 06, 2018.

doi: $10.21037 /$ tcr.2018.08.11

View this article at: http://dx.doi.org/10.21037/tcr.2018.08.11

The discovery of molecular subtypes of non-small cell lung cancer (NSCLC) with oncogenic driver mutations and translocations/rearrangements has led to successful development of targeted therapies and improvement in outcomes of advanced lung cancer patients. The frequency of these different subtypes and their clinical impact are illustrated in Figure 1A (1). Of these, drugs have already been approved by regulatory agencies for epidermal growth factor receptor (EGFR), anaplastic lymphoma kinase (ALK), ROS proto-oncogene 1 receptor tyrosine kinase (ROS1), and B-RAF proto-oncogene serine/threonine kinase (BRAF) targeted treatments. There are ongoing clinical trials for development of targeted therapies against other activated "driver oncogenes" in NSCLC, including MET proto-oncogene receptor tyrosine kinase (MET), ret proto-oncogene (RET), Erb-B2 receptor tyrosine kinase 2 (ERBB2), and neurotrophic receptor tyrosine kinase 1 (NTRK) among others.

ROS1 rearrangements were first reported in NSCLC in 2007 (2). Since then, ROS1 rearranged NSCLC has been described as a distinct molecular type in approximately $1-2 \%$ of patients with NSCLC $(2,3)$. It was the third clinically actionable subtype after EGFR-mutated and $A L K$ rearranged NSCLC to receive United States Food and Drug Administration (FDA) approval for a targeted therapy, the tyrosine kinase inhibitor (TKI) crizotinib.

The ROS1 locus is located on chromosome 6 and encodes for an orphan tyrosine kinase receptor, i.e., with no known ligand and biologic function in humans (4). ROS1 rearrangements/translocations lead to fusions of an intact ROS1 tyrosine kinase domain with partner genes, which are usually present on another chromosome (Figure 1B) $(5,6)$.
They can be detected in clinical samples via fluorescence in situ hybridization (FISH), immunohistochemistry (IHC), reverse transcription polymerase chain reaction (RTPCR), and next-generation sequencing (NGS) (2). So far, 14 different fusion partner genes have been identified in lung cancer patients, including CD74, SLC34A2, Syndecan 4 gene $(S D C 4)$, ezrin gene $(E Z R)$, fused in glioblastoma gene $(F I G)$, tropomyosin 3 gene (TPM3), leucine-rich repeats and immunoglobulin-like domains 3 gene (LRIG3), KDEL endoplasmic reticulum protein retention receptor 2 gene (KDELR2), coiled-coil domain containing 6 gene $(C C D C 6)$, moesin gene $(M S N)$, transmembrane protein 106B gene (TMEM106B), tumor protein D52 like 1 gene (TPD 52L1), clathrin heavy chain gene (CLTC), and LIM domain and acting binding 1 gene (LIMA1) $(2,5)$. Of these, the CD74-ROS1 fusion has been reported as the most common rearrangement in NSCLC. These fusion events lead to constitutive activation of the ROS1 kinase that drives cellular transformation and promotes survival and proliferation through downstream signaling via SHP-1/ SHP-2, JAK/STAT, PI3K/AKT/MTOR and MAPK/ERK pathways $(2,4,5,7)$.

With the discovery that lung cancers with ROS1 rearrangements are dependent on the driver oncogene, there was a natural interest in developing ROS1-targeted TKIs as a tailored treatment option for these patients. Given that ROS1-rearranged cancers form an undeniably small subset of patients with NSCLC, larger comparative trials are likely not going to be feasible. A number of phase I/II studies have been successfully performed however, demonstrating the utility of targeting this driver mutation and leading to a growing list of treatment options for this disease (Tables 1,2). 
A

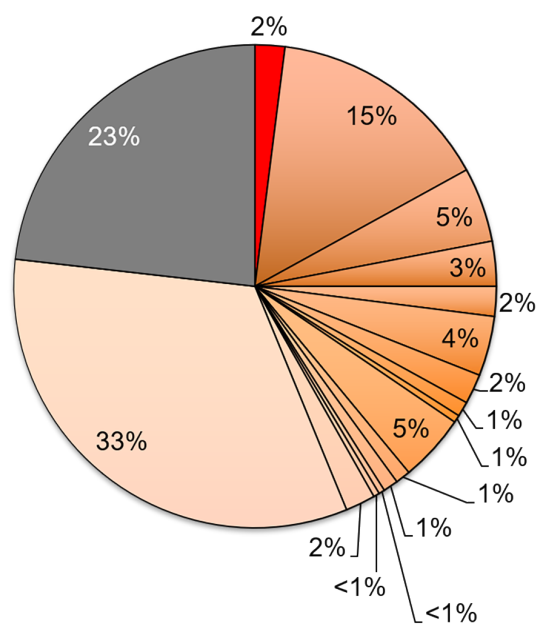

B

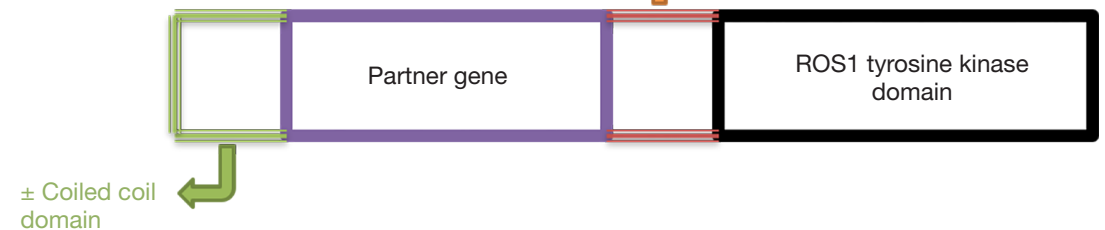

$\square$ ROS1 rearrangements

口EGFR mutations

$\square A L K$ rearrangements

$\square B R A F$ mutations

$\square M E T$ amplification (high

$\square$ MET exon 14 skipping

口ERBB2 mutations

$\square R E T$ rearrangements

口NTRK1 rearrangements

口NF1 mutations

口FGFR2/3/4

口FGFR2/3/4
mutations/rearrangements 口MAP2K1 mutations

$\square N R A S$ mutations

口HRAS mutations

口RIT1 mutations

$\square K R A S$ mutations

घUnknown/other

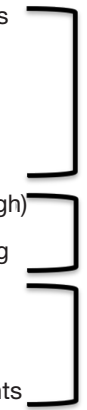

FDA approved

drugs

Emerging data

Potential future

targets
Evolving data
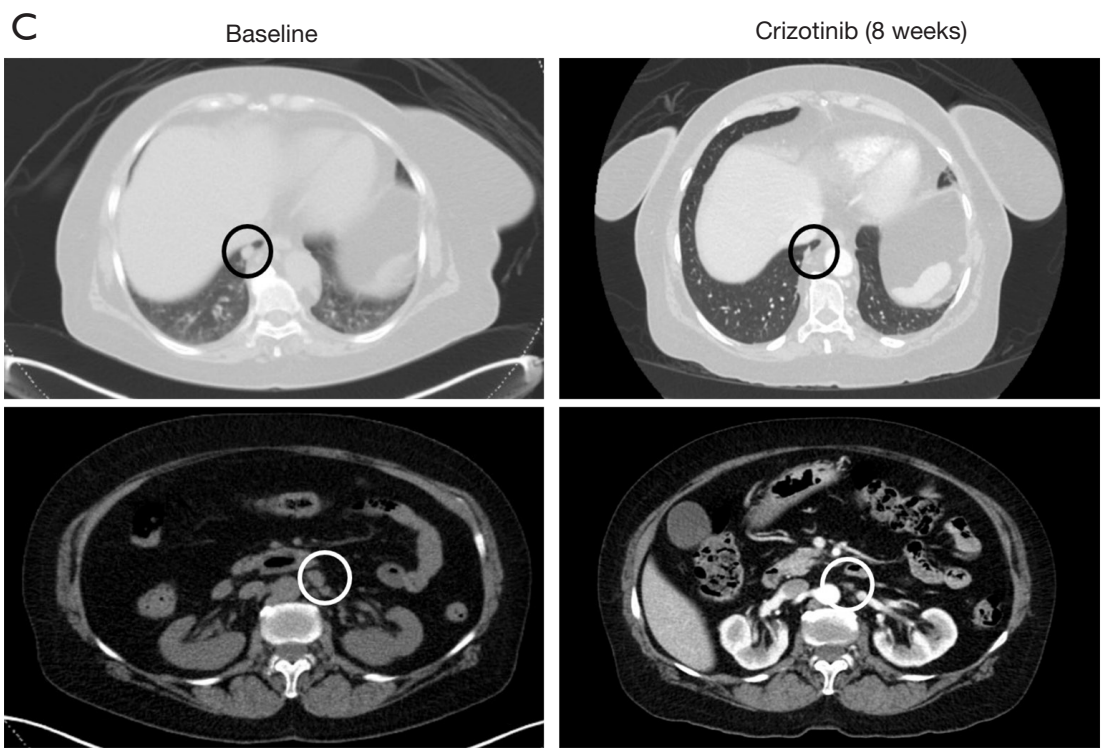

Figure 1 ROS1 rearrangements in context. (A) Frequency of molecular subtypes of non-small cell lung cancer of adenocarcinoma histology with a focus on ROS1 rearrangements; (B) representation of ROS1 partner fusion proteins. All constructors retain the ROS1 tyrosine kinase domain. ROS1 transmembrane and coiled-coil domains are variably present or absent in different fusions; (C) representative pre- and posttreatment images at 8 weeks in a patient with advanced lung cancer with a ROS1 rearrangement treated with first line crizotinib $250 \mathrm{mg}$ twice daily. Black circles indicate thoracic tumor burden; whole white circles indicate mesenteric lymphadenopathy. 
ROS1 and ALK tyrosine kinase domains share significant homology, including bindings sites for adenosine triphosphate (ATP) and crizotinib $(3,8)$. Although, ROS1 rearrangements and $A L K$ rearrangements are mutually exclusive $(3,5,17)$, they share similar clinicopathological features $(3,6)$. Both are generally seen in younger patients with light or never smoking history and have a preponderance for adenocarcinoma histology. However, their patterns of metastatic spread were recently found to be different (18). ROS1-rearranged NSCLC was described to have significantly lower rates of extra-thoracic and intracranial metastases at the time of diagnosis, as well as lower cumulative incidence of intracranial metastases. A subsequent single institution retrospective study has, however, questioned these findings by describing similar rates of intracranial metastases at diagnosis among patients with $A L K$ - and ROS1-rearranged lung cancers (19).

Given the considerable clinicopathological overlap and shared homology between the ROS1 and ALK tyrosine kinases, it should come as no surprise that crizotinib-a multitargeted MET/ALK/ROS1 inhibitor-was shown to have considerable clinical efficacy in ROS1-rearranged lung cancers. The global phase 1 study (PROFILE 1001) was amended to include ROS1-rearranged lung cancer in the expansion cohort, with a brisk overall response rate (ORR) of $72 \%$, disease control rate (DCR) of $90 \%$, and median progression-free survival (PFS) of 19.2 months, much as had been seen with $A L K$-rearranged tumors (8). On the basis of these striking results, crizotinib was granted full approval in the spring of 2016 by the FDA for treatment of advanced ROS1-rearranged lung cancer and remains to date the only approved treatment for this molecularly defined subset. Two subsequent studies from Europe have demonstrated PFS about half of that originally described for crizotinib in PROFILE 1001, in the 9-10-month range $(9,10)$. However, it should be noted that for both of these studies, the number of patients evaluated was low $(<30)$, and the EUROS 1 study was retrospective (Table 1). Later iterations of TKIsceritinib (12) and entrectinib (13) - -have both shown to be potent inhibitors in $A L K$-rearranged NSCLC and have also been shown to have overlap activity with ROS1-rearranged disease. Although phase I/II studies have suggested disease activity in crizotinib naïve cases (ORR $>70 \%$ and PFS $~ 19$ months), their activity seems to be considerably less so in the setting of crizotinib drug resistance. In comparison, lorlatinib-a highly potent, central nervous system (CNS)penetrant, and selective ALK and ROS1 TKI-was shown in a multicenter phase 1 trial to be active in crizotinib-resistant disease. Amongst the cohort of 12 patients with ROS1rearranged tumors and including seven crizotinib pretreated patients, an objective response was achieved by $6(50 \%)$ (14). Clinical activity seen with other TKIs in ROS1-rearranged NSCLC has also been described (Tables 1,2) $(15,16)$.

Although patients with ROS1-rearranged NSCLC have been shown to have variable rates of brain metastases at baseline as compared with $A L K$-rearranged disease, the brain remains a common and clinically relevant site of disease progression $(18,19)$. A recent study found crizotinib resistance mutations in $64 \%$ of non-intracranial specimens compared to $0 \%$ in three intracranial specimens (18). Lack of ROS1 resistance mutations in the CNS points to pharmacokinetic barriers as the underlying mode of drug failure, as crizotinib is known to have limited blood-brain barrier penetrance (20). TKIs that can penetrate the bloodbrain barrier may provide more durable disease responses. Ceritinib, entrectinib, and lorlatinib-all have better CNS penetration and demonstrable CNS activity (Tables 1,2). Although interpretation must be made with caution due to small numbers, they may soon supersede crizotinib by virtue of better prevention and control of recalcitrant CNS disease.

Data on the safety and tolerability of crizotinib in ROS1rearranged cohorts does not differ considerably from larger cohorts using this agent for $A L K$-rearranged disease. There were no treatment-related Common Terminology Criteria for Adverse Events (CTCAE) grade 4 or 5 events reported by Shaw et al. (8). The most common adverse events included mild visual impairment (described as transient dark-light adaptation adjustments) seen in $>80 \%$, peripheral edema $(>30 \%)$, and gastrointestinal side effects including nausea, vomiting, diarrhea, and constipation, which were all mostly grade 1 and seen in up to a third of the total cohort (8). Cross-trial comparisons suggest more frequent gastrointestinal toxicities with ceritinib (50-70\%), leading to dose reductions and adjustments $(12,21)$. Entrectinib and lorlatinib, on the other hand, seem to have a lower incidence of nausea, diarrhea, and constipation (10-20\%) although it is notable that lorlatinib was associated with an almost $40 \%$ incidence of peripheral neuropathy and cognitive adverse events that have not been noted in other studies of TKIs in this setting $(13,14)$.

Wu et al. have further expanded the armamentarium of evidence in this domain with the recent publication of the first prospective phase II international, single-arm, openlabel study of crizotinib in East Asian patients with ROS1rearranged advanced NSCLC (11). This trial studied 127 patients from China, Japan, South Korea, and Taiwan with locally advanced or metastatic NSCLC patients who had received 3 or fewer prior lines of systemic therapy; those with prior exposure to a ROS1- or ALK-directed therapy were excluded. ROS1 rearrangement status was assessed by 


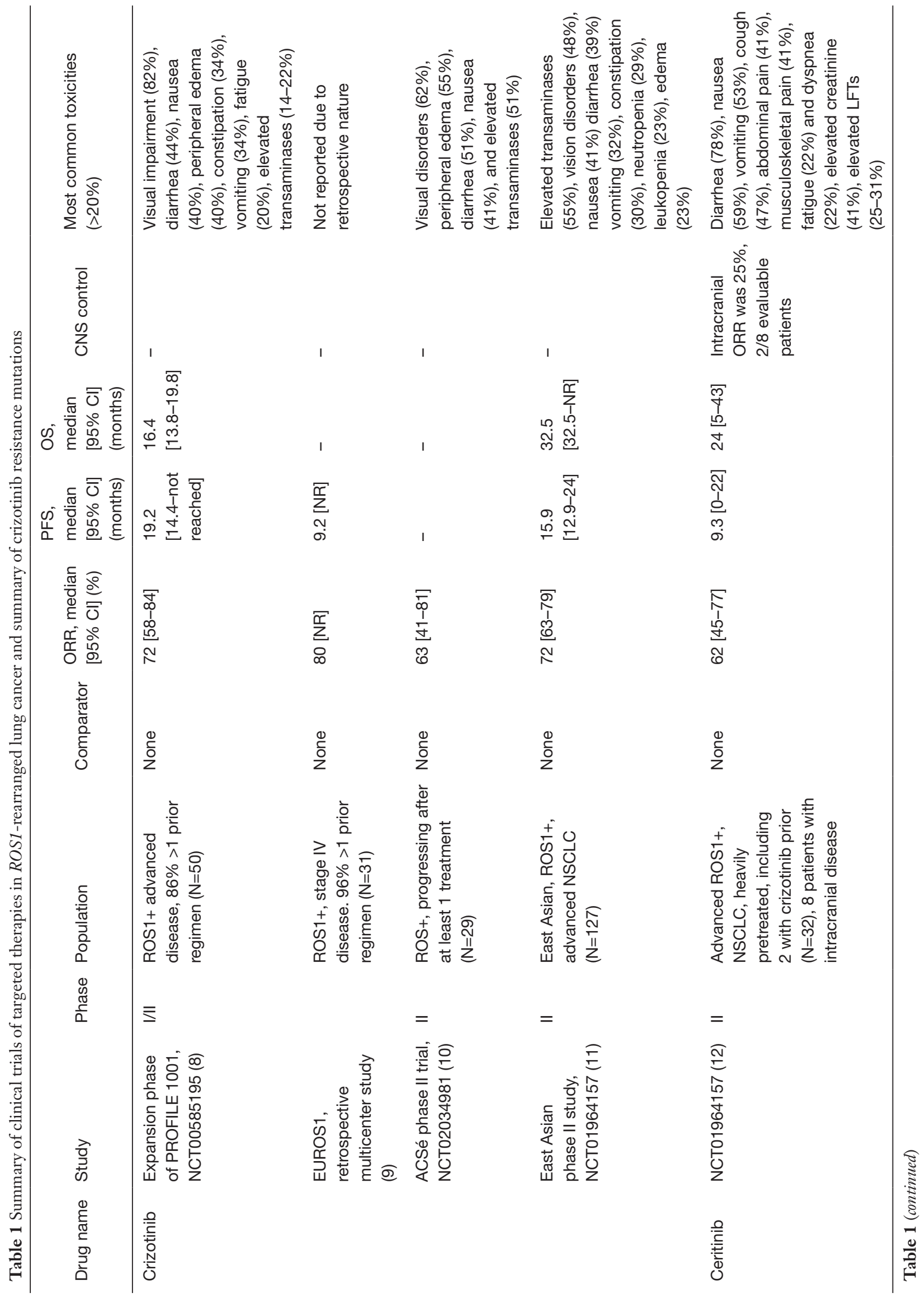




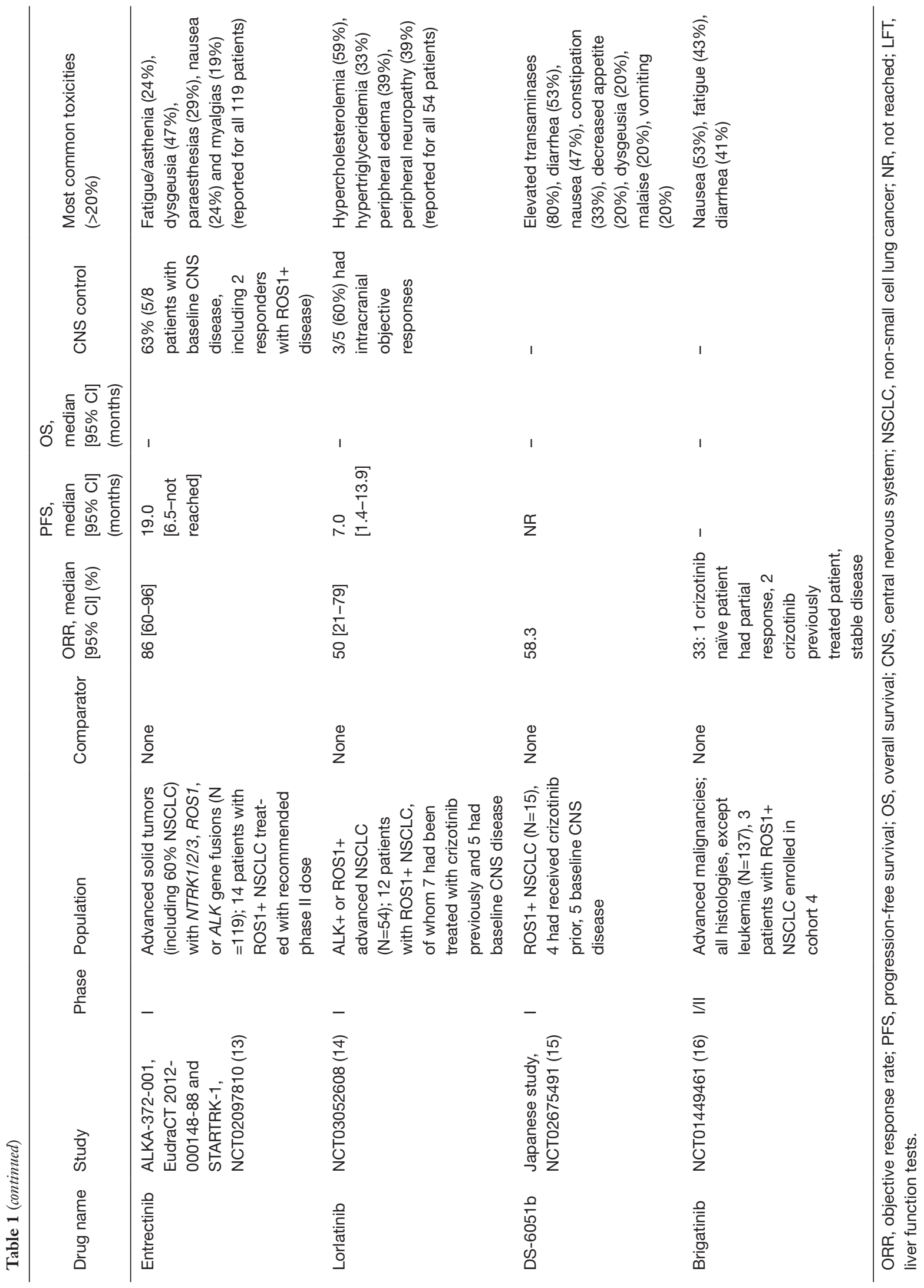


Table 2 Summary of crizotinib resistance mutations and drugs with pre-clinical or clinical activity in ROS1-rearranged lung cancer

\begin{tabular}{lll}
\hline $\begin{array}{l}\text { Putative ROS1 kinase domain crizotinib } \\
\text { resistance mutations }\end{array}$ & Drugs with preclinical activity & $\begin{array}{l}\text { Drugs with clinical response in case } \\
\text { reports }\end{array}$ \\
\hline G2032R & Repotrectinib, cabozantinib & Repotrectinib \\
D2033N & Lorlatinib, repotrectinib, cabozantinib & Cabozantinib \\
S1986Y/F & Lorlatinib & Lorlatinib \\
L2026M & Ceritinib, brigatinib, lorlatinib, repotrectinib, cabozantinib - \\
L1951R & Cabozantinib & - \\
\hline
\end{tabular}

validated RT-PCR using the AmoyDx ROS1 Gene Fusions Detection Kit at three regional laboratories. The patient population was similar in its characteristics to previously reported cohorts: most were younger than 65 years old (83.5\%), non-smokers (71.7\%), and had adenocarcinoma tumor histology (97.6\%). Most patients had been exposed to at least 1 prior line of therapy (81.1\%). ORR with crizotinib was found to be $71.7 \%$ (95\% CI: 63.0-79.3\%) with complete response in $13.4 \%$ patients. Responses were rapid in onset (median time to response of 1.9 months) and durable (median duration of response 19.7 months). Subgroup analysis showed that responses were maintained across all baseline characteristics (sex, age $<65$ or $>65$ years, smoking history, and number of prior lines of therapy both less than or more than or equal to 2). Median PFS was 15.9 months (95\% CI: 12.9-24.0 months). ORR was similar to high rates seen in the expansion cohort of the PROFILE 1001 trial (8) and preliminary findings of the two European prospective studies $(13,22)$. The PFS reported in this trial was less than reported in the expansion cohort of PROFILE 1001 (19.2 months). Crizotinib was generally well tolerated with no new safety signals identified. The most common adverse events included: elevated liver enzymes, vision disorder, nausea, diarrhea, and vomiting. CTCAE grade 3 and 4 drug-related adverse events were seen in $25.2 \%$ patients, with neutropenia and elevated liver enzymes being the most common ones. Patient-reported outcomes were also assessed and showed clinically meaningful improvements in the respiratory symptoms and expected deterioration of bowel habits. Within the limitations of a single-arm phase II study, it confirmed the clinical efficacy of crizotinib in ROS1rearranged lung cancer in this demographic and has led to regulatory approval of crizotinib in China, Japan, South Korea, and Taiwan. Although crizotinib showed response in both first and later-line settings, it would be hard to justify not using it as first line therapy in newly diagnosed patients with ROS1-rearranged NSCLC, given brisk and more durable efficacy with more limited toxicity compared to what might be expected with other established forms of palliative systemic therapy, i.e., chemotherapy, in this setting. As acknowledged by the authors themselves, a significant limitation of the study was that response of intracranial disease was not assessed. This is particularly relevant, given more limited CNS penetrance and intracranial efficacy with crizotinib (20).

The experience of on-label use of crizotinib in ROS1rearranged NSCLC patients in our own multidisciplinary clinic has been similar. We report here a case of a 66-year-old woman and life-long never smoker, who initially presented with shortness of breath. She was found to have extensive bilateral pulmonary embolism and incidentally noted to have diffuse supra-and sub-diaphragmatic lymphadenopathy along with a small right lower lobe lung lesion. Magnetic resonance imaging (MRI) of the brain showed no evidence of intracranial metastatic disease. Due to initial suspicion for a lymphoproliferative disorder, excisional lymph node biopsy was performed and revealed a poorly differentiated adenocarcinoma of lung primary with ROS1 rearrangement positive by FISH (84\% cells); comprehensive genomic profiling (Foundation One, Foundation Medicine, Cambridge, MA) confirmed a CD74-ROS1 fusion gene. The patient was started on palliative crizotinib $250 \mathrm{mg}$ twice daily, with repeat imaging 8 weeks later showing near complete response with massive regression of diffuse adenopathy and the primary right lung nodule (Figure 1C). At the time of this report nearly 11 months later, the patient continues to have a sustained response with limited toxicity, save mild fatigue and nausea and without need for dose reduction or interruption.

Although crizotinib has been associated with remarkable and durable clinical responses, resistance is an inevitable reality for patients and occurs through two succinct mechanisms: (I) "on target" mutations in crizotinib binding sites within the ROS1 tyrosine kinase domain, and (II) "off target" mechanisms including activation of bypass signaling pathways (i.e., EGFR, RAS and KIT) and phenotypic changes such as epithelial to mesenchymal transition $(18,23)$. 
"On target" crizotinib resistance mutations are thought to occur more frequently in ROS1- (approximately 50-60\%) compared to $A L K$ - (approximately 20-25\%) rearranged NSCLCs. However, these mutations have been described to involve a narrower segment of the tyrosine kinase domain in ROS1-rearranged NSCLC $(2,18)$. Table 2 describes the different ROS1 resistance mutations reported to date $(18,23)$. The most common crizotinib resistance mutation described is ROS1-G2032 R mutation. Other mutations include solvent front D2033N, S1986Y/F, gatekeeper L2026M, and L1951R. Various drugs have been tested against these resistance mutations using in vitro studies. Those with either preclinical activity or case reports of clinical efficacy against those mutations are described in Tables $1,2(2,18)$. Cabozantinib is a multi-targeted TKI that is thought to be effective for the majority of these resistance mutations. However, significant toxicities due to its non-selective mechanism of action have precluded its widespread use in this setting thus far (2). Lorlatinib is a highly potent and CNS-penetrant ALK/ROS1 inhibitor, which has in vitro activity against ROS1 rearrangements with $R O S 1-\mathrm{D} 2033 \mathrm{~N}$, S1986Y/F, and L2026M mutations. Based upon preclinical studies, its efficacy against ROS1-G2032R mutation may be limited and requires further investigation (2). Repotrectinib (formerly known as TPX-0005) has shown efficacy against ROS1-G2032R mutation (Table 2).

Important issues in the clinical management of patients with actionable genomic events such as ROS1 rearrangements include the optimal sequencing and utility of other treatment modalities, including cytotoxic chemotherapeutic agents and immune checkpoint inhibitors. In small retrospective studies, pemetrexed-based regimens had lower ORR (54-57\%) and a shorter PFS of 7-8 months (24) when compared with what has been seen in phase I-II studies with TKIs in this molecularly defined subset of advanced NSCLC. Of note, compared to other oncogenic driver mutations in NSCLC, ROS1-rearranged lung cancers may be associated with better responses to chemotherapy.

Phase I-II clinical trials, retrospective reviews and, small case series on patients with crizotinib resistance mutations have provided significant insight and led to improvements in the outcomes of patients with ROS1-rearranged NSCLC. Although the infrequency of this driver mutation will likely preclude direct comparisons between agents, the brisk and durable efficacy of ROS1-directed TKIs has led to widespread use of these agents in the upfront management of advanced stage disease in this subset of patients. The role of immune checkpoint inhibitors is still not clear and merits further study, although the experience with immune monotherapy in NSCLC with other actionable oncogenic driver mutations has been disappointing to date. Current efforts include attempting to determine if there is synergistic antitumor activity with combined immunotherapy and targeted therapy (25). Additionally, high rates of CNS progression associated with crizotinib use and the inevitable development of crizotinib resistance mean that significant challenges exist as we try to transform the long-term outcomes of patients with this disease. Similar to the use of osimertinib and alectinib as initial therapy in EGFR-mutated and $A L K$-rearranged advanced NSCLC, respectively, it remains a worthy cause to develop advanced generation CNS-penetrant ROS1-directed therapies that will have efficacy against the most common crizotinib resistance mutations and can be propelled into the frontline setting for these patients.

\section{Acknowledgments}

We acknowledge Thomas Anderson, MD in the Department of Radiology at Beth Israel Deaconess Medical Center for help in selection of radiographic images.

Funding: This work was funded in part through a National Institutes of Health (NIH)/National Cancer Institute (NCI) grant R37CA218707 (to DB Costa).

\section{Footnote}

Provenance and Peer Review: This article was commissioned and reviewed by the Section Editor Wei Xu (Division of Respiratory Disease, Department of Geriatrics, the First Affiliated Hospital of Nanjing Medical University, Nanjing, China).

Conflicts of Interest: DB Costa has consulting fees and honoraria from Pfizer, Astra-Zeneca and ARIAD/Takeda. The other authors have no conflicts of interest to declare.

Ethical Statement: The authors are accountable for all aspects of the work in ensuring that questions related to the accuracy or integrity of any part of the work are appropriately investigated and resolved.

Open Access Statement: This is an Open Access article distributed in accordance with the Creative Commons Attribution-NonCommercial-NoDerivs 4.0 International License (CC BY-NC-ND 4.0), which permits the noncommercial replication and distribution of the article with the strict proviso that no changes or edits are made and the original work is properly cited (including links to both the formal publication through the relevant DOI and the license). 
See: https://creativecommons.org/licenses/by-nc-nd/4.0/.

\section{References}

1. Shea M, Costa DB, Rangachari D. Management of advanced non-small cell lung cancers with known mutations or rearrangements: latest evidence and treatment approaches. Ther Adv Respir Dis 2016;10:113-29.

2. Lin JJ, Shaw AT. Recent Advances in Targeting ROS1 in Lung Cancer. J Thorac Oncol 2017;12:1611-25.

3. Bergethon K, Shaw AT, Ou SH, et al. ROS1 rearrangements define a unique molecular class of lung cancers. J Clin Oncol 2012;30:863-70.

4. Acquaviva J, Wong R, Charest A. The multifaceted roles of the receptor tyrosine kinase ROS in development and cancer. Biochim Biophys Acta 2009;1795:37-52.

5. Gainor JF, Shaw AT. Novel targets in non-small cell lung cancer: ROS1 and RET fusions. Oncologist 2013;18:865-75.

6. Takeuchi K, Soda M, Togashi Y, et al. RET, ROS1 and ALK fusions in lung cancer. Nat Med 2012;18:378-81.

7. Davies KD, Doebele RC. Molecular pathways: ROS1 fusion proteins in cancer. Clin Cancer Res 2013;19:4040-5.

8. Shaw AT, Ou SH, Bang YJ, et al. Crizotinib in ROS1rearranged non-small-cell lung cancer. N Engl J Med 2014;371:1963-71.

9. Mazières J, Zalcman G, Crinò L, et al. Crizotinib therapy for advanced lung adenocarcinoma and a ROS1 rearrangement: results from the EUROS1 cohort. J Clin Oncol 2015;33:992-9.

10. Moro-Sibilot D, Faivre L, Zalcman G, et al. Crizotinib in patients with advanced ROS1-rearranged non-small cell lung cancer (NSCLC). Preliminary results of the ACSé phase II trial. J Clin Oncol 2015;33:8065.

11. Wu YL, Yang JC, Kim DW, et al. Phase II Study of Crizotinib in East Asian Patients With ROS1-Positive Advanced Non-Small-Cell Lung Cancer. J Clin Oncol 2018;36:1405-11.

12. Lim SM, Kim HR, Lee JS, et al. Open-Label, Multicenter, Phase II Study of Ceritinib in Patients With Non-SmallCell Lung Cancer Harboring ROS1 Rearrangement. J Clin Oncol 2017;35:2613-8.

13. Drilon A, Siena S, Ou SI, et al. Safety and Antitumor Activity of the Multitargeted Pan-TRK, ROS1, and ALK Inhibitor Entrectinib: Combined Results from Two Phase I Trials (ALKA-372-001 and STARTRK-1). Cancer Discov 2017;7:400-9.

14. Shaw AT, Felip E, Bauer TM, et al. Lorlatinib in nonsmall-cell lung cancer with ALK or ROS1 rearrangement: an international, multicentre, open-label, single-arm firstin-man phase 1 trial. Lancet Oncol 2017;18:1590-9.
15. Fujiwara Y, Takeda M, Yamamoto N, et al. Safety and pharmacokinetics of DS-6051b in Japanese patients with non-small cell lung cancer harboring ROS1 fusions: a phase I study. Oncotarget 2018;9:23729-37.

16. Gettinger SN, Bazhenova LA, Langer CJ, et al. Activity and safety of brigatinib in ALK-rearranged non-small-cell lung cancer and other malignancies: a single-arm, openlabel, phase 1/2 trial. Lancet Oncol 2016;17:1683-96.

17. Lin JJ, Ritterhouse LL, Ali SM, et al. ROS1 Fusions Rarely Overlap with Other Oncogenic Drivers in NonSmall Cell Lung Cancer. J Thorac Oncol 2017;12:872-7.

18. Gainor JF, Tseng D, Yoda S, et al. Patterns of Metastatic Spread and Mechanisms of Resistance to Crizotinib in ROS1-Positive Non-Small-Cell Lung Cancer. JCO Precis Oncol 2017;2017. doi: 10.1200/PO.17.00063.

19. Patil T, Smith DE, Bunn PA, et al. The incidence of brain metastases in stage IV ROS1-rearranged non-small cell lung cancer and rate of central nervous system progression on crizotinib. J Thorac Oncol 2018. [Epub ahead of print].

20. Costa DB, Kobayashi S, Pandya SS, et al. CSF concentration of the anaplastic lymphoma kinase inhibitor crizotinib. J Clin Oncol 2011;29:e443-5.

21. Dagogo-Jack I, Shaw AT. Expanding the Roster of ROS1 Inhibitors. J Clin Oncol 2017;35:2595-7.

22. Michels S, Gardizi M, Schmalz P, et al. MA07.05 EUCROSS: A European Phase II Trial of Crizotinib in Advanced Adenocarcinoma of the Lung Harboring ROS1 Rearrangements - Preliminary Results. J Thorac Oncol 2017;12:S379-80.

23. Song A, Kim TM, Kim DW, et al. Molecular Changes Associated with Acquired Resistance to Crizotinib in ROS1-Rearranged Non-Small Cell Lung Cancer. Clin Cancer Res 2015;21:2379-87.

24. Chen YF, Hsieh MS, Wu SG, et al. Efficacy of Pemetrexed-Based Chemotherapy in Patients with ROS1 Fusion-Positive Lung Adenocarcinoma Compared with in Patients Harboring Other Driver Mutations in East Asian Populations. J Thorac Oncol 2016;11:1140-52.

25. Moya-Horno I, Viteri S, Karachaliou N, et al. Combination of immunotherapy with targeted therapies in advanced non-small cell lung cancer (NSCLC). Ther Adv Med Oncol 2018;10:1758834017745012.

Cite this article as: Sehgal K, Patell R, Rangachari D, Costa DB. Targeting ROS1 rearrangements in non-small cell lung cancer with crizotinib and other kinase inhibitors. Transl Cancer Res 2018;7(Suppl 7):S779-S786. doi: 10.21037/ tcr.2018.08.11 